\title{
Electrochemical Properties of 1,3-Dimethylimidazolium Dicyanamide, Bis(trifluoromethanesulfonyl)imide and Dimethylphosphate Ionic Liquids
}

\author{
Yong Zheng ${ }^{1, *}$, Yongjun Zheng ${ }^{1}$, Qian Wang ${ }^{2}$, Huichao Lv $^{1}$, Zhen Wang ${ }^{1}$ \\ ${ }^{1}$ College of Chemical and Environmental Engineering, Anyang Institute of Technology, Anyang \\ 455000, P. R. China \\ ${ }^{2}$ State Key Laboratory of Multiphase Complex Systems, Institute of Process Engineering, Chinese \\ Academy of Sciences, Beijing 100190, P. R. China \\ *E-mail: yzheng83@126.com
}

Received: 8 August 2021 / Accepted: 17 September 2021 / Published: 10 October 2021

\begin{abstract}
1,3-dimethylimidazolium-based ionic liquids show promise as electrolytes because of their special cation structure. However, their electrochemical properties remain less understood. Thus, 1,3dimethylimidazolium dicyanamide, bis(trifluoromethanesulfonyl)imide and dimethylphosphate were chosen as representative 1,3-dimethylimidazolium-based ionic liquids in this work. The electrical conductivity and molar conductivity of these ionic liquids were measured and calculated from 303.15 to $353.15 \mathrm{~K}$, which were well fitted with empirical equations. Meanwhile, the corresponding electrochemical window and differential capacitance were also obtained by cyclic voltammetry and impedance measurements, respectively. On the basis of experimental data and theoretical calculations, the relationships between the electrochemical properties and molecular structures of the ionic liquids were assessed in detail. These ionic liquids have higher electrical conductivity and wider electrochemical windows than many other 1-alkyl-3-methylimidaozlium-based ionic liquids with the same anions. These phenomena are probably ascribed to the higher structural symmetry, stronger cation-anion interaction and smaller size of the 1,3-dimethylimidazolium cation. Moreover, 1,3-dimethylimidazolium dicyanamide has a higher dissociation of ions, while 1,3-dimethylimidazolium bis(trifluoromethanesulfonyl)imide and dimethylphosphate have a lower ionic dissociation. 1,3dimethylimidazolium cations are prone to be adsorbed onto the Pt plate electrodes when the cationanion interaction is lower. It is hoped that the present work will provide important electrochemical research results for the future application of these ionic liquids.
\end{abstract}

Keywords: 1,3-dimethylimidazolium; ionic liquid; electrical conductivity; electrochemical window; differential capacitance 
(C) 2021 The Authors. Published by ESG (www.electrochemsci.org). This article is an open access article distributed under the terms and conditions of the Creative Commons Attribution license (http://creativecommons.org/licenses/by/4.0/). 